\title{
Study of Brain Activation Using Electroencephalographic Technique for Performing Short-Term Memory Tests
}

\author{
Edgard Souza Fernandes Filho', Thiago Vinícius de Oliveira Lima', \\ Diêgo Lucas Ramos e Silva1, Milton Vieira Costa ${ }^{2}$, Euclides Mauricio Trindade Filho ${ }^{2 *}$ \\ ${ }^{1}$ Health Sciences Centre, State University of Health Sciences of Alagoas-UNCISAL, Maceió, Brazil \\ ${ }^{2}$ Department of Physiology, State University of Health Sciences of Alagoas-UNCISAL, Maceió, Brazil \\ Email: ${ }^{*}$ emtfilho@gmail.com
}

Received 10 January 2016; accepted 16 February 2016; published 19 February 2016

Copyright (C) 2016 by authors and Scientific Research Publishing Inc.

This work is licensed under the Creative Commons Attribution International License (CC BY).

http://creativecommons.org/licenses/by/4.0/

(c) (i) Open Access

\begin{abstract}
Objective: This study aimed to compare the cortical topographic mapping while performing cognitive activities of standardized short-term memory. Materials and Methods: The sample consisted of 30 individuals of both gender. Each individual participant of the survey was subjected to a short-term memory test for each sense. To carry out the EEG record, we used an electroencephalograph with 20 electrodes. The stimulus for the acquisition of short-term memory has always been made up of five items from different semantic classes. Results: The posterior right quadrant had a higher percentage of gamma rhythm during the tests of most senses. Conclusion: It was concluded that the right back quadrant has a higher gamma rhythms percentage during tests which involve somesthetic, olfactory and gustatory memory. On the other hand, the predominance of a gamma rhythm percentage in any quadrant when the auditory and visual memory was stimulated was not observed in this study.
\end{abstract}

\section{Keywords}

EEG, Short-Term Memory, Cognition

\section{Introduction}

The organization of human memory consists of at least two systems with different lengths: a short-term memory (STM) and a long term memory (LTM). The formation of memories starts primarily with the acquisition of in-

\footnotetext{
${ }^{*}$ Corresponding author.
}

How to cite this paper: Filho, E.S.F., Lima, T.V.O., Silva, D.L.R., Costa, M.V. and Filho, E.M.T. (2016) Study of Brain Activation Using Electroencephalographic Technique for Performing Short-Term Memory Tests. World Journal of Neuroscience, 6, 37-42. http://dx.doi.org/10.4236/wjns.2016.61005 
formation in sensory systems (vision, hearing, touch, etc.) as stimuli [1].

The short-term memory stores the recent events by continuous neural activity because STM persists for just a minute [2]. The short-term memory has a limited supply of information that can seize only about 7 to 12 items at a time. The short-term memory has a limited supply of information that can retain only about 7 to 12 items at a time.

The electroencephalogram (EEG) can be characterized in a simplified way like the registration of a mixture of rhythmic voltage fluctuations similar to sine waves which represent the variations of the differences in voltage between two points on the skull surface. These fluctuations are interpreted taking into consideration the frequency variations and amplitude [3].

The EEG frequency variations are grouped into four categories: alpha, beta, theta and delta [3]. More recently, some authors have suggested that frequencies between 25 - $45 \mathrm{~Hz}$, which they termed gamma rhythm, accounted electrical correlates of cognitive activities execution. Thus, the record of a rhythm in a determined cortical area while performing a mental activity suggests that cortical area would be responsible for the processing of mental activity.

Although the systematic presence of a particular frequency enables the diagnosis of a rhythm, it is normally observed frequency waves superposition across the spectrum to build maps with percentages with various frequencies in different cranial areas through mathematical techniques. Such a technique known as brain mapping allowed the quantification of the presence of different rhythms in places where the electrodes were positioned [3] [4].

Therefore, it becomes interesting to identify cortical areas involved in the processing of short term memory using procedure with high temporal resolution such as cortical topographic mapping.

\section{Materials and Methods}

This study was approved by the Research Ethics Committee (REC) of UNCISAL with the protocol number of 447,856 in 2013.

This was a study of observational and cross-sectional. The survey was conducted in the laboratory of Neuroscience and Behavior at the University of Health Sciences of Alagoas (UNCISAL).

The sample consisted of 30 individuals of either sex aged between 20 and 40 years, of which 18 (60\%) were male and 12 (40\%) were female. These volunteers were recruited by the researchers among college students, Most (50\%) were studying Medicine, other volunteers were students from other courses in the health area. It included individuals of either gender aged 20 to 40 years. It excluded the ones who were making use of psychotropic drugs or who showed behavioral changes.

To carry out the EEG record, the electroencephalograph EMSA brand, were used (320 serial model) with 20 electrodes, which were placed on the volunteers scalp according to the international 10 - 20 system. To place the electrodes a conductive bentonite paste was used. Signs of brain electrical activity experienced a sampling rate of $256 \mathrm{~Hz}$ and were filtered for more in the range 0.1 Hz. The EEG signals were amplified, digitized and the information from brain mapping was analyzed. All information has been stored on CDs.

The volunteers were recruited according to criteria for inclusion and exclusion. After the clear explanation of what would be carried out during the research and the application of informed consent, some personal information were raised, because the use of some types of recreational drugs or not, could interfere in the research results.

Each individual participant in the research was subjected to a test of short-term memory intended for each sense.

Electroencephalographic examinations were conducted always in morning period between 8:00 and 12:00. Each session consisted, initially, of five minutes of waking basal record. Then the Electroencephalographic record was continued simultaneously with:

Presentation of the stimulus to short-term memory for 30 seconds;

Ten seconds of distracting tasks (from 1 to reverse form 10);

Evocation of short-term memory for 20 seconds;

This sequence was repeated in the same individual using stimuli for each sense: Visual, auditory, Olfactory, Gustatory and Somesthetic.

The stimulus for the acquisition of short-term memory has always been made up of five items of different semantic classes. 
Visual: orange; hammer; car; knife; shirt (represented by pictures on a computer screen).

Hearing: Nail; fork; pants; apple; School (spoken through a tape recorder).

Somesthetic: Cotton; pencil; die; Screw; fabric (placed sequentially always on right hand).

Olfaction: Perfume, vinegar, lemon, coffee and acetone (placed in a $2 \mathrm{ml}$ beaker and smelled two centimeter away from the nostrils).

Taste: Salt; sugar; cinnamon; apple; wine (placed in small quantities on the anterior surface of the tongue).

The sample size was set at 30 volunteers being calculated by statistical program Bio-estat version 5.0, taking into account a minimum difference between the averages of 3 treatments, a standard deviation of 3 error; the number of treatments equal to 5; the power of 0.95 test and an alpha of 0.05 in a bilateral test.

The data were presented as an average and standard deviation, including tables. The comparison between the relative powers (percentage of the gamma rhythm frequencies) between the period of control and the stimulus period as well as between the control period and the recall period was performed using the Student $\mathrm{t}$ test. The 0.05 significance level was accepted to reject the null hypothesis.

\section{Results}

Quantitative analysis of topographic mapping, the study of the sense of vision showed that there was no significant difference in the percentage of the gamma rhythm when compared to control situation with the visual stimulation situation. On the other hand, significant difference was observed $(p=0.039)$ when compared to control situation with the situation of evocation of visual short-term memory. In this case it was observed an increase in the percentage of the gamma rhythm of the posterior right quadrant during the recall phase of the sense of vision (Table 1).

During the study of auditory short-term memory, there was no significant difference in the percentage of gamma rhythm when compared to control situation with the auditory stimulus and short-term memory recall auditory (Table 2).

In the evaluation of the somesthetic sense, it was observed a higher percentage of the gamma rhythm on the posterior right quadrant $(p=0.025)$ during the somesthetic stimulus when compared to the control. On the other hand, there was no significant difference during the recall of short-term memory somesthetic (Table 3).

In the evaluation of the sense of smell, there was a statistically significant presence $(p=0.031)$ of a higher percentage of the gamma rhythm in the area of the posterior right quadrant when compared to control situation with the olfactory stimulus situation. On the other hand, there was no significant difference when compared to control situation with short term memory recall of the situation (Table 4).

The analysis of short term memory of the sense of taste, showed the presence of a significant higher percentage of the gamma rhythm in the anterior right quadrants $(p=0.011)$, left anterior $(p=0.022)$ and posterior right $(p=0.002)$ when compared to control situation with stimulus presentation. However, during the evocation of short-term memory gustatory, it was observed significant increase in gamma rhythm in all quadrants (Table 5).

Table 1. Gamma rhythm percentage on brain quadrants during the control of the vision sensory stimulation and during the evocation of short-term memory.

\begin{tabular}{ccccc}
\hline & AL $(\mathrm{X} \pm \mathrm{SD})$ & AR $(\mathrm{X} \pm \mathrm{SD})$ & PR $(\mathrm{X} \pm \mathrm{SD})$ & $27.37 \pm 1.787$ \\
\hline Control & $27.307 \pm 1.749$ & $27.173 \pm 1.492$ & $27.833 \pm 1.842$ & $26.76 \pm 0.868$ \\
Sense of vision & $27.923 \pm 1.979$ & $27.22 \pm 1.498$ & $27.497 \pm 1.635$ & $27.513 \pm 1.256$ \\
Evocation & $27.737 \pm 1.605$ & $27.2 \pm 1.494$ & 27001 \\
\hline
\end{tabular}

Legend: SD—Standard Deviation, AL—Anterior Left, AR—Anterior Right, PL—Posterior Left, PR—Posterior Right.

Table 2. Gamma rhythm percentage on brain quadrants during control, sensory stimulation of the hearing and during the evocation of short-term memory.

\begin{tabular}{ccccc}
\hline & AL $(\mathrm{X} \pm \mathrm{SD})$ & AR $(\mathrm{X} \pm \mathrm{SD})$ & PR $(\mathrm{X} \pm \mathrm{SD})$ & $27.37 \pm 1.787$ \\
\hline Control & $27.307 \pm 1.749$ & $27.173 \pm 1.492$ & $27.65 \pm 1.766$ & $27.19 \pm 1.400$ \\
Sense of hearing & $27.47 \pm 1.576$ & $27.327 \pm 1.308$ & $26.937 \pm 1.145$ & $27.223 \pm 1.389$ \\
Evocation & $27.557 \pm 1.803$ & $27.82 \pm 1.584$ & 27.001 \\
\hline
\end{tabular}

Legend: SD—Standard Deviation, AL—Anterior Left, AR—Anterior Right, PL—Posterior Left, PR—Posterior Right. 
Table 3. Gamma rhythm percentage on brain quadrants during control, sensory stimulation of touch and during the evocation of short-term memory.

\begin{tabular}{ccccc}
\hline & AL $(\mathrm{X} \pm \mathrm{SD})$ & AR $(\mathrm{X} \pm \mathrm{SD})$ & $\mathrm{PR}(\mathrm{X} \pm \mathrm{SD})$ & $\mathrm{PL}(\mathrm{X} \pm \mathrm{SD})$ \\
\hline Control & $27.307 \pm 1.749$ & $27.173 \pm 1.492$ & $27.37 \pm 1.787$ & $26.927 \pm 1.001$ \\
Somesthetic & $26.963 \pm 3.483$ & $27.907 \pm 1.969$ & $27.753 \pm 1.804$ & $27.72 \pm 1.706$ \\
Evocation & $27.393 \pm 1.361$ & $27.863 \pm 1.735$ & $27.2 \pm 1.157$ & $27.277 \pm 1.382$ \\
\hline
\end{tabular}

Legend: SD—Standard Deviation, AL—Anterior Left, AR—Anterior Right, PL—Posterior Left, PR—Posterior Right.

Table 4. Gamma rhythm percentage on brain quadrants during control, sensory stimulation of smell and during the evocation of short-term memory.

\begin{tabular}{ccccc}
\hline & AL $(\mathrm{X} \pm \mathrm{SD})$ & AR $(\mathrm{X} \pm \mathrm{SD})$ & $\mathrm{PR}(\mathrm{X} \pm \mathrm{SD})$ & $27.37 \pm 1.787$ \\
\hline Control & $27.307 \pm 1.749$ & $27.173 \pm 1.492$ & $275.55 \pm 1.656$ & $27.637 \pm 1.621$ \\
Sense of smell & $27.57 \pm 1.438$ & $27.333 \pm 1.798$ & $27.683 \pm 1.933$ & $27.48 \pm 1.533$ \\
Evocatio & $27.783 \pm 1.729$ & $27.33 \pm 1.868$ & $\mathrm{SD}$ \\
\hline
\end{tabular}

Legend: SD—Standard Deviation, AL—Anterior Left, AR—Anterior Right, PL—Posterior Left, PR—Posterior Right.

Table 5. Gamma rhythm percentage on brain quadrants during the control of taste and sensory stimulation during the evocation of short-term memory.

\begin{tabular}{ccccc}
\hline & AL $(\mathrm{X} \pm \mathrm{SD})$ & $\mathrm{AR}(\mathrm{X} \pm \mathrm{SD})$ & $\mathrm{PR}(\mathrm{X} \pm \mathrm{SD})$ & $\mathrm{PL}(\mathrm{X} \pm \mathrm{SD})$ \\
\hline Control & $27.307 \pm 1.749$ & $27.173 \pm 1.492$ & $27.37 \pm 1.787$ & $26.927 \pm 1.001$ \\
Sense of taste & $28.02 \pm 1.667$ & $28.087 \pm 1.894$ & $28.04 \pm 1.886$ & $28.14 \pm 1.765$ \\
Evocation & $28.07 \pm 2.07$ & $28.01 \pm 1.93$ & $28.497 \pm 1.943$ & $28.107 \pm 2.145$ \\
\hline
\end{tabular}

Legend: SD—Standard Deviation, AL—Anterior Left, AR—Anterior Right, PL—Posterior Left, PR-Posterior Right.

\section{Discussion}

High-frequency cortical activities (gamma rhythm) mainly $40 \mathrm{~Hz}$, have been reported in various parts of the brain cortex reflecting higher cognitive processes such as attention, perception and higher cognitive processes [5]. In our analysis, it was determined that the gamma rhythm would be the one with frequencies between 26.2 and $32 \mathrm{~Hz}$ which is used in most studies that use the frequency range to record the gamma rhythm between 25 and $45 \mathrm{~Hz}$ [6]-[8].

In animal studies, using the EEG with small electrodes, it was observed the existence of the gamma rhytmh in auditory memory, visual, olfactory, and motor somesthetic in several of species animals [9]. Similarly, our study has shown high activity in the gamma rhythm during the somesthetic and olfactory stimuli, as well as for gustatory stimuli. On the other hand, a significant percentage of gamma rhythm was not observed during the auditory and visual stimuli, in the latter was only observed a significant percentage of gamma rhythm during the recall period. This result is interesting, since in another study in which various cognitive tasks during the EEG record were applied was observed a much less intense gamma rhythm increase during the hearing task using music, compared to other cognitive tasks performed in the study [10].

Therefore we believe that there could be some brain quadrant with a predominant percentage of gamma rhythm if music were to be used music as an auditory stimulation in our study, whereas in other study it has been shown with the use of EEG that the N400 component typically related to semantic processing verbal language is found in a similar way during the semantic information processing nonverbal (music) [11].

However, areas such as the hippocampus activation while listening to music can be related to the music advantage in the acquisition of memory compared to the auditory stimulus by verbal language [12] [13].

In the present study, despite the fact that nouns were used during the auditory stimulus, there was no significant presence of gamma rhythm in any of the cerebral quadrants of the cortical topographic mapping. These findings are discordant from those observed in another study which confirmed a significant presence of the 
gamma rhythm with more posterior cerebral topography during the auditory stimulus involving nouns [14].

As in the auditory stimulus, a significant difference on the percentage of the gamma rhythm when control situation and visual stimulus are compared was not observed. Such finding does not confirm what was observed in the Sauseng et al. study [15] which during investigations of brain oscillatory activity using EEG to predict short term memory capacity through visual stimuli, showed a significant increase in the gamma rhythm in the back right site of the brain during visual memory tasks.

In this study, the posterior right quadrant was where the presence of gamma rhythm predominated during the presentation of the somesthestic, olfactory and taste stimuli. This finding lacks information in the literature. However, in another study using functional magnetic resonance imaging (fMRI) to investigate brain activation during cognitive assessment with a list of semantically related and unrelated words, it was observed that the regions adjacent to the intra-parietal sulcus and right cerebral hemisphere of the lateral occipital were the regions more activated [16].

In this study, there was a significant higher percentage of gamma rhythm in posterior right quadrant, left and right anterior when compared to control condition with the condition of gustatory stimulation. In his study, Pereira, Reis and Magalhães [17], reported that while investigating the mapping cortical brain through Functional Magnetic Resonance Imaging of the paradigms sensorimotor of the Homunculus of Penfield focal activation of the mobility of the tongue in the posterior region of the right hemisphere was observed. Thus, Despite being scarce in the literature studies with similar findings as our study, we believe that tasting (one of the tongue functions) is a sense in which the gamma rhythm percentage dominates the posterior right quadrant, this fact was statistically shown as the quadrants where cerebral activation during gustative stimulus were compared.

Our hypothesis about the no predominance in any quadrant occurred due to the fact that the gustatory stimulation occurred in clear nostrils. Thus the percentage of gamma rhythm may have increased in most brain quadrants as there have been a possibility of the olfactory stimuli influence during the activity involving tasting as there is an association between olfaction and tasting [18].

It is noteworthy that there are no studies investigating the presence of gamma rhythm when compared to a waking state with evocation of short-term memory involving the sense organs. However, in our study it wasn't observed statistically significant difference when control condition was compared to the short-term memory recall condition of the majority of the senses. We can infer that during brain activation through cognitive activities there are few cortical areas that may present gamma rhythm when short-term memory is evoked after performing these activities. This may be so because the distracting task did not allow the information gathered during the short-term memory tests were prolonged for long-term memory.

\section{Conclusion}

The result of our study allows us to speculate that the reverberating circuits responsible for several short-term memories are concentrated at the back right quadrant. However, in this study the predominance of gamma rhythm percentage was not observed in any brain quadrant when auditory and visual memories stimulated.

\section{Funding}

Edgard Souza Fernandes Filho who held a scholarship by the Foundation of Research Protection-FAPEAL.

\section{Competing Interests}

The authors report no conflicts of interest.

\section{References}

[1] Graeff, F.G. and Brandão, M.L. (1997) Neurobiology of Mental Illness. Lemos Publisher, São Paulo.

[2] Berner, R.M. (2004) Physiology. Elsevier, Rio de Janeiro.

[3] Duffy, L.A. (1999) Cerebral Topographic Mapping. Ateneu, São Paulo.

[4] Braga, N.I.O. (1993) The Importance of Topographic Mapping in Neurology. In: Nitrini, R. and Machado, L.R., Eds., Conduct in Neurology, São Paulo.

[5] Ihara, A., Hirata, M., Sakihara, K., Izumi, H., Takahashi, Y., Kono, K., Imaoka, H., Osaki, Y., Kato, A., Yoshimine, T. and Yorifugi, S. (2003) Gamma-Band Desynchronization in Language Areas Reflects Syntactic Process of Words. Neuroscience Letters, 339, 135-138. http://dx.doi.org/10.1016/S0304-3940(03)00005-3 
[6] Morillon, B., Chauvel, C.L., Arnal, L.H., Bénar, C.G. and Giraud, A.L. (2012) Asymmetric Function of Theta and Gamma Activity in Syllable Processing: An Intra-Cortical Study. Frontiers in Psychology, 19, 248. http://dx.doi.org/10.3389/fpsyg.2012.00248

[7] Zaeyen, E.J.B. (2005) Consistent Application to EEG to Investigate Characteristics of Evoked Potentials and Middle Latency Auditory. Master Degree Dissertation, Federal University of Rio de Janeiro, Rio de Janeiro.

[8] Willems, R.M., Oostenveld, R. and Hagoort, P. (2008) Early Decreases in Alpha and Gamma Band Power Distinguish Linguistic from Visual Information during Spoken Sentence Comprehension. Brain Research, 1219, 78-90. http://dx.doi.org/10.1016/j.brainres.2008.04.065

[9] Engel A.K. and Singer, W. (2001) Temporal Binding and the Neural Correlates of Sensory Awareness. Trends in Cognitive Sciences, 5, 16-25. http://dx.doi.org/10.1016/S1364-6613(00)01568-0

[10] Fitzgibbon, S.P., Pope, K.J., Mackenzie, L., Clark, C.R. and Willoughby, J.O. (2004) Cognitive Tasks Augment Gamma EEG Power. Clinical Neurophysiology, 115, 1802-1809. http://dx.doi.org/10.1016/j.clinph.2004.03.009

[11] Koelsch, S., Kasper, E., Sammler, D., Schulze, K., Gunter, T. and Friederici, A.D. (2004) Music, Language and Meaning: Brain Signatures of Semantic Processing. Nature Neuroscience, 7, 302-307. http://dx.doi.org/10.1038/nn1197

[12] Koelsch, S. (2010) Towards a Neural Basis of Music-Evoked Emotions. Trends in Cognitive Sciences, 14, $131-137$. http://dx.doi.org/10.1016/j.tics.2010.01.002

[13] Rocha, V.C. and Boggio, P.S. (2013) Music by a Neuroscientific Perspective. Per Musi, 27, 132-140. http://dx.doi.org/10.1590/S1517-75992013000100012

[14] Khader, P. and Rosler, F. (2004) EEG Power and Coherence Analysis of Visually Presented Nouns and Verbs Reveals Left Frontal Processing Differences. Neuroscience Letters, 354, 111-114. http://dx.doi.org/10.1016/j.neulet.2003.10.016

[15] Sauseng, P., Klimesch, W., Heise, K.F., Gruber, W.R., Holz, E., Karlim, A.A., Glennon, M., Gerloff, C., Birbaumer, N. and Hummel, F.C. (2009) Brain Oscillatory Substrates of Visual Short-Term Memory Capacity. Current Biology, 19, 1846-1852. http://dx.doi.org/10.1016/j.cub.2009.08.062

[16] Batistuzzo, M.C. (2013) Brain Activation Associated with Verbal Episodic Memory in OCD by Means of Functional Magnetic Resonance Imaging. Ph.D. Dissertation, University of São Paulo, São Paulo.

[17] Pereira, J.R., Reis, A.M. and Magalhães, Z. (2013) Functional Neuroanatomy: Anatomy of the Activatable Areas in the Usual Paradigms in Functional MRI. Portuguese Acta Medica, 16, 107-116.

[18] Seiberling, K.A. and Conley, D.B. (2004) Aging and Olfactory and Taste Function. Otolaryngologic Clinics of North America, 37, 1209-1228. http://dx.doi.org/10.1016/j.otc.2004.06.006 